\title{
TWO-DIMENSIONAL SCHEME FOR CONVECTION-DIFFUSION WITH LINEAR PRODUCTION
}

\author{
Y. H. Chen and Tony W. H. Sheu \\ Department of Naval Architecture and Ocean Engineering, National Taiwan \\ University, 73 Chou-Shan Rd., Taipei, Taiwan, Republic of China.
}

In this article we consider the scalar transport governed by the convection-diffusion equation with linear production in two dimensions. The underlying idea in the development of a steady discretization scheme is to incorporate the analytical solution, obtained within a one-dimensional context, into the formulation. The method adopted here features exponential character in the weighted coefficients of the matrix equation, which generates a scheme with high accuracy. The analysis is followed by extending this discretization scheme to solve the convection-diffusion-production equation with a source term. In this article we present an analytical validation study of the method applied to two classes of model equations. Having verified that the method applies equally well for a transport scalar in either case with constant or variable flow velocity, we employ the one-dimensional scheme in the predictor and corrector steps to obtain the two-dimensional solution in an alternatingdirection-implicit fashion. The result obtained for the test considered in this article follows the trend of analytic data.

\section{INTRODUCTION}

The linear scalar advection-diffusion equation has long been examined as the simplest prototype for Navier-Stokes equations. Sufficient detail can be gained from analysis of this equation at considerably less expense. This explains why this equation has been a subject of academic importance in the area of fluid dynamics and heat transfer over the last few decades. It is fundamentally difficult to solve this equation due to the presence of potentially destabilizing first-order derivative terms in the equation [1]. Numerical pathologies immediately appear as derivatives of this sort are approximated by centered schemes. Although the use of different upwinding remedies has achieved some success in suppressing oscillatory solutions, the need to add artificial damping terms, implicitly or explicitly, to the formulation has placed limitations on the computing techniques. It is the objective of the current study to fix this problem by designing a stable flux discretization scheme which retains a high level of accuracy at relatively low added computing expense.

The Helmholtz equation represents another classical problem in the field of applied mathematics. This elliptic equation is used to describe propagating and evanescent waves in acoustic fields [2]. Other application examples include electro-

Received 15 June 1999; accepted 27 August 1999.

The research reported in this work was financially supported by grant NSC 88-2611-E-002-02 5 of the Republic of China.

Address correspondence to Prof. Tony W. H. Sheu, Institute of Naval Architecture, National Taiwan University, 73 Chou-Shan Rd., Taipei, Taiwan, Republic of China. E-mail: sheu@, indy.na.ntu.edu.tw 


\section{NOMENCLATURE}

\begin{tabular}{|c|c|c|c|}
\hline $\mathrm{E}, \mathrm{W}, \mathrm{S}, \mathrm{N}$ & $\begin{array}{l}\text { east, west, south, north of node } \mathrm{P} \\
\text { shown in Figure } 1\end{array}$ & $v$ & $\begin{array}{l}\text { velocity of the flow along the } \\
\text { direction }\end{array}$ \\
\hline$g$ & $\begin{array}{l}\text { specified value of } \phi \text { along the } \\
\text { boundary }\end{array}$ & $\begin{array}{l}\beta \\
\Gamma\end{array}$ & $\begin{array}{l}\beta=\frac{1}{2 K}\left(\mathrm{u} \pm \sqrt{u^{2}-4 K R^{2}}\right) \\
\text { the boundary of } \Omega\end{array}$ \\
\hline$h$ & uniform grid size & $\Phi$ & a scalar which serves as the \\
\hline$K$ & diffusivity of the fluid & & dependent variable \\
\hline $\mathrm{P}$ & centroid of an element & $\Omega$ & the physical domain under \\
\hline$R$ & strength of the production term & & investigation \\
\hline$u$ & velocity of the flow along the $x$ & $\nabla$ & gradient operator \\
\hline & direction & $\nabla \cdot$ & divergence operator \\
\hline
\end{tabular}

magnetic waves and diffusion problems in semiconductors. In the literature, research efforts have particularly been paid to the scheme stability because of the potential loss of ellipticity with increasing wave numbers in the propagation region.

As a subject of fundamental importance, it is the objective of the present study to simulate the generalized equation for the above two classes of equations, namely, the convection-diffusion-productio $n$ equation. Difficulties involved in solving steady-state solutions of this model equation arise from the skew-symmetric convective term and the positive production term in the equation, since they have potentially destabilizing effects. The intention to gain insight into the behavior inherent in the solution is, thus, certainly worthy of consideration.

In the next section we describe the working equation. This is followed by the description of the discretization method presented in the article. The underlying idea in the development of an advection-diffusion-productio $\mathrm{n}$ scheme is to incorporate the analytic information into the formulation. We also show that for steady problems our scheme is applicable to homogeneous/inhomogeneous problems with variable velocities. This is followed by extending the analysis to two dimensions employing the developed one-dimensional flux discretization scheme in each step of the alternating-direction-implicit solution algorithm. The introduced discretization errors become known by the modified equation analysis. We then present results for three test problems to benchmark the scheme presented here. Conclusions are drawn in the final section.

\section{MODEL EQUATION}

In this article we consider a linear advection-diffusion-production equation. For this steady-state study, we employ a linear source to model production in the domain $\Omega$.

$$
K \nabla^{2} \phi-u \nabla \phi \pm R^{2} \phi=0
$$

In the above equation, $\nabla^{2}$ is the Laplacian operator, and $\nabla$ is the gradient operator. In Eq. (1), $K$ denotes the diffusion coefficient, assumed to be constant and positive. For simplicity, $u$ is the constant flow velocity prescribed a priori in the formulation. As for $R^{2}$, it represents the strength of the linear source. The 
boundary of the open domain $\Omega$, namely, $\Gamma$, is taken as being piecewise smooth. To close the elliptic differential equation (1), we consider here only the Dirichlet-type boundary condition for purposes of presentation:

$$
\phi=g \quad \text { on } \quad \Gamma
$$

\section{NUMERICAL ANALYSIS}

\section{One-Dimensional Linear Equation}

In an isotropic medium where $K$ is assumed to be constant over the entire domain, we consider first the constant flow case so as to facilitate description of the method. The equation under investigation is given by

$$
K \frac{d^{2} \phi}{d x^{2}}-u \frac{d \phi}{d x} \pm R^{2} \phi=0
$$

By performing a change of variable given by

$$
\phi=\Phi \exp \left(\frac{u x}{2 K}\right)
$$

a standard Helmholtz equation for $\Phi$ is obtained as

$$
K \frac{d^{2} \Phi}{d x^{2}}+\left(\frac{u^{2}}{4 K} \pm R^{2}\right) \phi=0
$$

In light of the analytic solution of $\Phi$ which takes the form

$$
\Phi=a \exp \left(\sqrt{\frac{u^{2}}{4 K^{2}} \mp \frac{R^{2}}{K}} x\right)+b \exp \left(-\sqrt{\frac{u^{2}}{4 K^{2}} \mp \frac{R^{2}}{K}} x\right)
$$

we are led to know that propagating or evanescent waves coexist in the solution. form:

By substituting Eq. (6) into (4), the general solution of (3) takes the following

$$
\phi=a \exp \left[\frac{1}{2 K}\left(u+\sqrt{u^{2} \mp 4 K R^{2}}\right) x\right]+b \exp \left[\frac{1}{2 K}\left(u-\sqrt{u^{2} \mp 4 K R^{2}}\right) x\right]
$$

It can be inferred from Eq. (7) that the solution of (3) consists of two types of solutions. The nature of the solution either varies exponentially at a composite rate or propagates exponentially at a modulated pace. Let

$$
\begin{aligned}
& \beta_{1}=\frac{1}{2 K}\left(u+\sqrt{u^{2} \mp 4 K R^{2}}\right) \\
& \beta_{2}=\frac{1}{2 K}\left(u-\sqrt{u^{2} \mp 4 K R^{2}}\right)
\end{aligned}
$$


The general solution to Eq. (1) can be rewritten as

$$
\phi=a \exp \left(\beta_{1} x\right)+b \exp \left(\beta_{2} x\right)
$$

We now turn to determining $a$ and $b$ in Eq. (7). Referring to Figure 1, the solution $\phi(x)$ is expressed as the sum of $\phi_{1}(x) \phi_{\mathrm{W}}$ and $\phi_{2}(x) \phi_{\mathrm{E}}$ :

$$
\phi(x)=\phi_{1}(x) \phi_{\mathrm{W}}+\phi_{2}(x) \phi_{\mathrm{E}}
$$

The solution $\phi$ is sought in a domain $\Omega$, with closed boundary surface $\Gamma$, upon which the following constraint conditions apply:

$$
\begin{aligned}
\phi_{1}(h) & =0 \\
\phi_{1}(-h) & =1 \\
\phi_{2}(h) & =1 \\
\phi_{2}(-h) & =0
\end{aligned}
$$

Subject to the above four conditions, we can easily derive the functional expressions of $\phi_{1}$ and $\phi_{2}$ as follows:

$$
\begin{aligned}
& \phi_{1}(x)=\frac{\exp \left(\beta_{1} h+\beta_{2} x\right)-\exp \left(\beta_{2} h+\beta_{1} x\right)}{2 \sinh \left[(h / K) \sqrt{u^{2} \pm 4 K R^{2}}\right]} \\
& \phi_{2}(x)=\frac{\exp \left(\beta_{1} x-\beta_{2} h\right)-\exp \left(\beta_{2} x-\beta_{1} h\right)}{2 \sinh \left[(h / K) \sqrt{u^{2} \pm 4 K R^{2}}\right]}
\end{aligned}
$$

Given the above derivation, $\phi_{\mathrm{P}}$ is obtained as

$$
\phi_{\mathrm{P}}=\phi(0)=\phi_{1}(0) \phi_{\mathrm{W}}+\phi_{2}(0) \phi_{\mathrm{E}}
$$

Substituting $\phi_{1}$ and $\phi_{2}$, given by Eqs. (12)-(13), into Eq. (14), we can obtain the discrete representation of Eq. (3) at point P:

$$
A_{\mathrm{P}} \phi_{\mathrm{P}}+A_{\mathrm{W}} \phi_{\mathrm{W}}+A_{\mathrm{E}} \phi_{\mathrm{E}}=0
$$

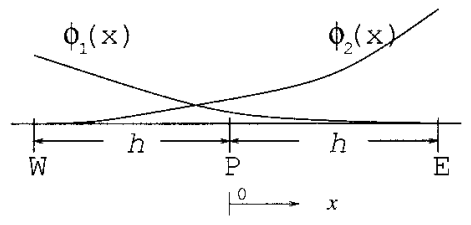

Figure 1. Illustration of solution $\phi$ at points $\mathrm{P}, \mathrm{E}, \mathrm{W}$ in a domain with $\Delta x=h$. 
where

$$
\begin{aligned}
& A_{\mathrm{P}}=-1 \\
& A_{\mathrm{W}}=\frac{\exp \left(\beta_{1} h\right)-\exp \left(\beta_{2} h\right)}{2 \sinh \left[(h / K) \sqrt{u^{2} \pm 4 K R^{2}}\right]} \\
& A_{\mathrm{E}}=\frac{\exp \left(-\beta_{2} h\right)-\exp \left(-\beta_{1} h\right)}{2 \sinh \left[(h / K) \sqrt{u^{2} \pm 4 K R^{2}}\right]}
\end{aligned}
$$

By employing the identity given by $\cosh (\beta h / 2)=\frac{1}{2}[\exp (\beta h / 2)+\exp (-\beta h / 2)]$, the weighting coefficients shown in Eqs. (17), (18) are rewritten as

$$
\begin{aligned}
A_{\mathrm{W}} & =\frac{\exp (u h / 2 K)}{2 \cosh \left[(h / K) \sqrt{u^{2} \pm 4 K R^{2}}\right]} \\
A_{\mathrm{E}} & =\frac{\exp (-u h / 2 K)}{2 \cosh \left[(h / K) \sqrt{u^{2} \pm 4 K R^{2}}\right]}
\end{aligned}
$$

The resulting tri-diagonal system of equations can be efficiently solved by the Thomas direct solution solver [3].

\section{Convection-Diffusion-Production Problem with a Source Term}

In this article we also consider the transport equation with a linear production $S(x)$ :

$$
K \frac{d^{2} \phi}{d x^{2}}-u \frac{d \phi}{d x} \pm R^{2} \phi+S(x)=0
$$

Let $\phi^{*}$ and $\phi$ be particular and numerical solutions of (21), respectively. The new variable $\hat{\phi}$, defined by

$$
\hat{\phi}=\phi-\phi^{*}
$$

satisfies

$$
K \frac{d^{2} \hat{\phi}}{d x^{2}}-u \frac{d \hat{\phi}}{d x} \pm R^{2} \hat{\phi}=0
$$

The idea behind the construction of the perpendicular solution $\phi^{*}$ is that the analytic derivation just presented can be applied directly to solving Eq. (23). By applying the same procedure as that considered in the previous section, the 
following equation is derived from the use of Eq. (22):

$$
A_{\mathrm{P}} \phi_{\mathrm{P}}+A_{\mathrm{W}} \phi_{\mathrm{W}}+A_{\mathrm{E}} \phi_{\mathrm{E}}=S_{u}
$$

where

$$
S_{u}=A_{\mathrm{P}} \phi_{\mathrm{P}}^{*}+A_{\mathrm{W}} \phi_{\mathrm{W}}^{*}+A_{\mathrm{E}} \phi_{\mathrm{E}}^{*}
$$

We now turn to solving the particular solution $\phi^{*}$. Let $\phi^{*}$ be approximated through Taylor series expansion with respect to $x_{\mathrm{P}}$. Provided that the Taylor series expansion is terminated at the polynomial order $N, \phi^{*}$ is given as

$$
\phi^{*}=\sum_{n=0}^{N} \gamma_{n}\left(x-x_{\mathrm{P}}\right)^{n}
$$

Having approximated $\phi^{*}$ as shown in Eq. (26), we can easily derive

$$
\begin{aligned}
& \frac{d \phi^{*}}{d x}=\sum_{n=1}^{N} n \gamma_{n}\left(x-x_{\mathrm{P}}\right)^{n-1} \\
& \frac{d^{2} \phi^{*}}{d x^{2}}=\sum_{n=2}^{N} n(n-1) \gamma_{n}\left(x-x_{\mathrm{P}}\right)^{n-2}
\end{aligned}
$$

In a similar fashion, we can also approximate $S(x)$ by virtue of Taylor series expansion as follows:

$$
S(x)=\sum_{n=1}^{N} \frac{S^{(n)}\left(x_{\mathrm{P}}\right)}{n !}\left(x-x_{\mathrm{P}}\right)^{n} .
$$

By substituting equations (22), (26)-(28) into

$$
K \frac{d^{2} \hat{\phi}}{d x^{2}}-u \frac{d \hat{\phi}}{d x} \pm R^{2} \hat{\phi}+S(x)=0
$$

we can obtain the expressions of $\gamma_{N}, \gamma_{N}-1$, and $\gamma_{n}-2(n=N, \ldots, 2)$ as follows:

$$
\begin{gathered}
\gamma_{N}=\frac{-S^{(N)}\left(x_{\mathrm{P}}\right)}{\left( \pm R^{2}\right) N !} \\
\gamma_{N-1}=\frac{-1}{(N-1) !}\left[\frac{u S^{(N)}\left(x_{\mathrm{P}}\right)}{\left( \pm R^{2}\right)^{2}}+\frac{S^{(N-1)}\left(x_{\mathrm{P}}\right)}{\left( \pm R^{2}\right)}\right] \\
\gamma_{n-2}=\frac{1}{ \pm R^{2}}\left[n(n-1)(1-K) \gamma_{n}-1-\frac{S^{(n-1)}\left(x_{\mathrm{P}}\right)}{(n-2) !}\right] \quad n=N, \ldots, 2
\end{gathered}
$$

This completes the one-dimensional formulation. 


\section{Convection-Diffusion-Production Scheme in Two Dimensions}

We now turn to extending the scheme development to analyze the following equation:

$$
-K\left(\phi_{x x}+\phi_{y y}\right)+u \phi_{x}+v \phi_{y} \pm 2 R^{2} \phi=0
$$

In the above, $u$ and $v$ represent the velocity components along the $x$ and $y$ directions, respectively. Other coefficients involve $K$ and $R^{2}$, which denote the diffusion coefficient and the production coefficient, respectively. For illustrative purposes, all these values are assumed to be constant throughout. For simplicity, we consider Eq. (34), which is subject to the Dirichlet-type boundary condition

$$
\phi=g \quad \text { on } \quad \partial \Omega
$$

We choose to follow the idea of alternating direction implicit (ADI) scheme to solve Eq. (34). In the course of operator splitting, calculation of the solution from Eq. (34) is accomplished in the following two steps.

Predictor step:

$$
u \phi_{x}^{*}-K \phi_{x x}^{*} \pm R^{2} \phi^{*}=f^{*}
$$

where

$$
f^{*}=-v \phi_{y}^{k}+K \phi_{y y}^{k} \mp R^{2} \phi^{k}
$$

Corrector step:

$$
v \phi_{y}^{k+1}-K \phi_{y y}^{k+1} \pm R^{2} \phi^{k+1}=f^{k+1}
$$

where

$$
f^{k+1}=-u \phi_{x}^{*}+K \phi_{x x}^{*} \mp R^{2} \phi^{*}
$$

In each step of the predictor-corrector calculation, we apply the nodally exact scheme given in the previous section to obtain the solutions. The numerical model was run using the two-step iterative solver, with a convergence criterion expressed by $\left[\phi^{k+1}-\phi^{k} / \phi^{k+1}\right]<\varepsilon$. In this study, the tolerance $\varepsilon$ is set equal to $10^{-10}$. As the convergence criterion is reached, the two-step solution is exactly the same as that computed from the following five-point stencil equation:

$$
A_{\mathrm{W}} \phi_{\mathrm{W}}+A_{\mathrm{E}} \phi_{\mathrm{E}}+A_{\mathrm{N}} \phi_{\mathrm{N}}+A_{\mathrm{S}} \phi_{\mathrm{S}}+A_{\mathrm{P}} \phi_{\mathrm{P}}=0
$$




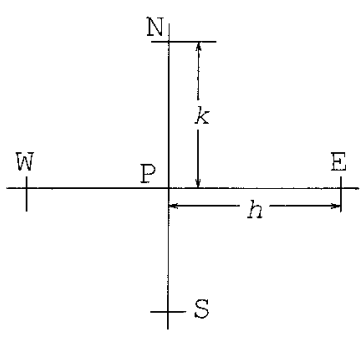

Figure 2. Definition of nodal points E, W, S, N, P in the two-dimensional analysis.

The coefficients at nodes E, W, S, N, and P, shown schematically in Figure 2, are as follows:

$$
\begin{aligned}
& A_{\mathrm{W}}=\frac{\exp (u h / 2 K)}{2 h^{2}} \\
& A_{\mathrm{E}}=\frac{\exp (-u h / 2 K)}{2 h^{2}} \\
& A_{\mathrm{S}}=\frac{\exp (v k / 2 K)}{2 k^{2}} \\
& A_{\mathrm{N}}=\frac{\exp (-v k / 2 K)}{2 k^{2}} \\
& A_{\mathrm{P}}=-\frac{\cosh \left[(h / 2 K) \sqrt{u^{2}-4 K R^{2}}\right]}{h^{2}}-\frac{\cosh \left[(k / 2 K) \sqrt{v^{2}-4 K R^{2}}\right]}{k^{2}}
\end{aligned}
$$

In order to shed light on the operator-splitting approximation error added into the two-dimensional analysis, we will conduct a modified equation analysis [4]. Substituting Taylor-series expressions into the five-point-stencil finite-difference equation, involving $\phi_{i, j}, \phi_{i \pm 1, j}$, and $\phi_{i, j \pm 1}$ nodal points, we obtain the following modified equation after some algebra:

$$
\begin{aligned}
K\left(\phi_{x x}-\right. & \left.\phi_{y y}\right)-u \phi_{x}-v \phi_{y}+2 R^{2} \phi \\
= & \left(-\frac{h^{2} u^{2} R^{2}}{24 K^{2}}-\frac{k^{2} v^{2} R^{2}}{24 K^{2}}+\frac{h^{2} R^{4}}{12 K}+\frac{k^{2} R^{4}}{12 K}\right) \phi \\
& +\left(-\frac{h^{2} u^{2}}{8 K}-\frac{h^{4} u^{4}}{384 K^{3}}\right) \phi_{x x}+\left(-\frac{k^{2} v^{2}}{8 K}-\frac{k^{4} v^{4}}{384 K^{3}}\right) \phi_{y y} \\
& +\left(-\frac{h^{2} u^{3}}{24 K^{2}}-\frac{h^{4} u^{5}}{1920 K^{4}}\right) \phi_{x}+\left(-\frac{k^{2} v^{3}}{24 K^{2}}-\frac{k^{4} v^{5}}{1920 K^{4}}\right) \phi_{y} \\
& -\left(\frac{h^{2} K}{12}+\frac{h^{4} u^{2}}{96 K}+\frac{h^{6} u^{4}}{4680 K^{4}}\right) \phi_{x x x x}-\left(\frac{k^{2} K}{12}+\frac{k^{4} v^{2}}{96 K}+\frac{k^{6} v^{4}}{4680 K^{4}}\right) \phi_{y y y y}
\end{aligned}
$$


As the above equation shows, it is clear that the consistency property that is necessary to obtain a convergent solution is satisfied as grid size $h$ approaches zero.

\section{COMPUTED RESULTS}

\section{Constant-Flow Analysis without Source Term}

The analysis is conducted in a domain bounded by two ends, $x=0$ and $x=1$. Subject to analytical data $\phi(0)=g_{0}$ and $\phi(1)=g_{1}$ at the two ends, the analytic solution takes the following form:

$$
\begin{aligned}
g_{0} \exp (u x / 2 K) \sin \left[\sqrt{\left(R^{2} / K\right)-\left(u^{2} / 4 K^{2}\right)}(1-x)\right] \\
\left.+g_{1} \exp \llbracket u(x-1) / 2 K\right] \sin \left[\sqrt{\left(R^{2} / K\right)-\left(u^{2} / 4 K^{2}\right)} x\right]
\end{aligned}
$$

For this constant-flow analysis, simulations were performed on two sets of data $(u, K, R)=(2,1,5)$ and $(20,1,2)$. Calculations were performed at uniformly spaced points with a resolution of $h=0.1$. As Figures 3 and 4 , which plot the computed results against spatial coordinate, indicate, the match of the analytic solution with the computed solutions is strikingly good for both cases. This demonstrates the integrity of the scheme and its performance.

\section{Variable-Flow Analysis without Source Term}

The success of applying our proposed scheme to a model linear convectiondiffusion-production equation motivated the analysis conducted for the case with variable coefficients. The case we considered was with $K=x^{2} / 2, u=x$, and

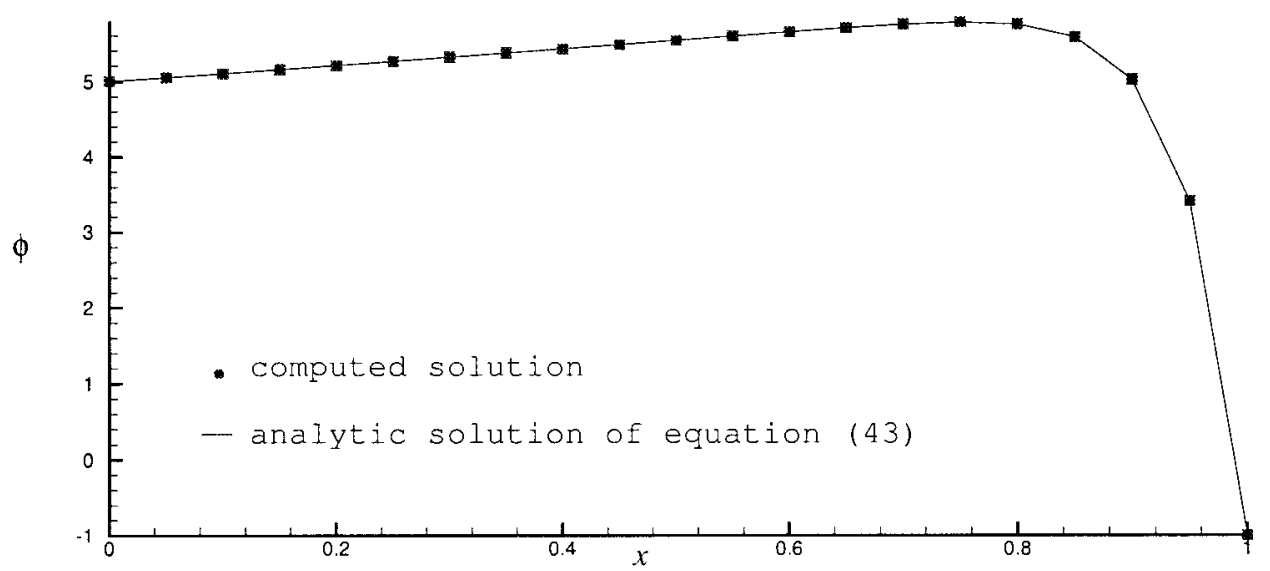

Figure 3. Comparison of computed and analytic solutions for the case $(u, K, R)=(20,1,2)$ described in the results section. 


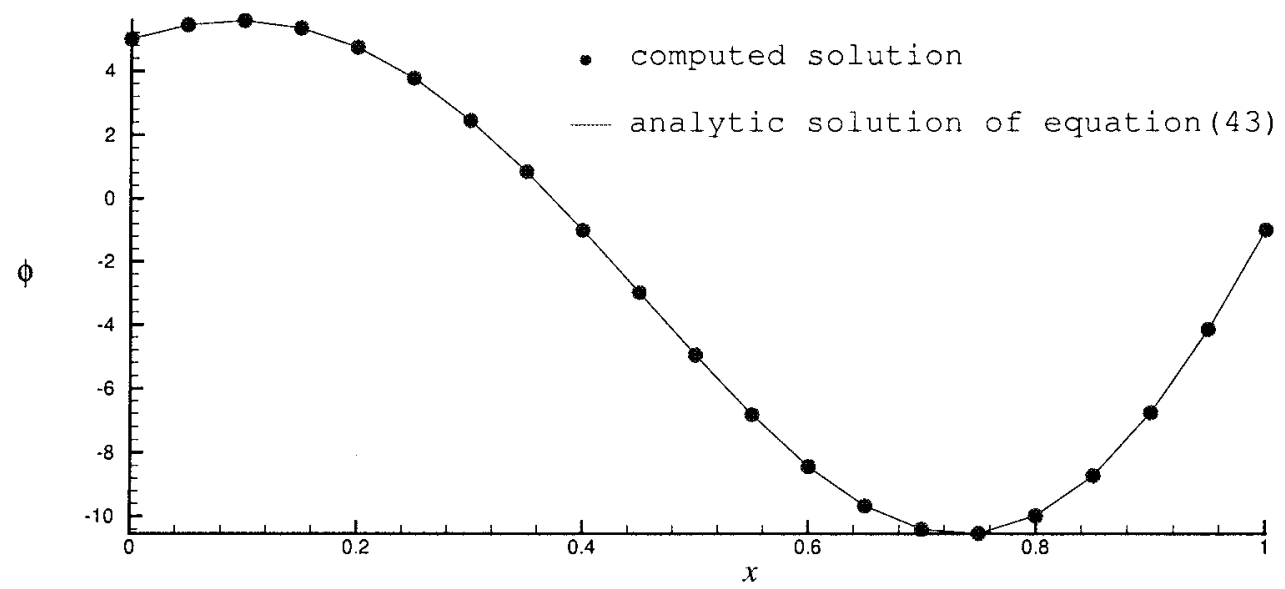

Figure 4. Comparison of computed and analytic solutions for the case $(u, K, R)=(2,1,5)$ described in the results section.

$R=1$. In the domain, defined as $0 \leq x \leq 1$, our calculation was performed in uniform meshes with resolution $h=0.1,0.05$, and 0.025. As Figure 5 shows, computed solutions approach analytic solution, $\phi=x^{2}$, as the grids kept being refined. This demonstrated the applicability of our scheme to simulate the variable convection-diffusion-production problem.

\section{Convection-Diffusion-Production Equation with a Source Term}

Having validated the applicability of our proposed scheme to simulate the convection-diffusion-production scheme, we considered the problem with $(u, K, R)$ $=(6,1,2)$ and a source term given by $S(x)=-2\left(3 x^{2}+1\right)$. In the domain

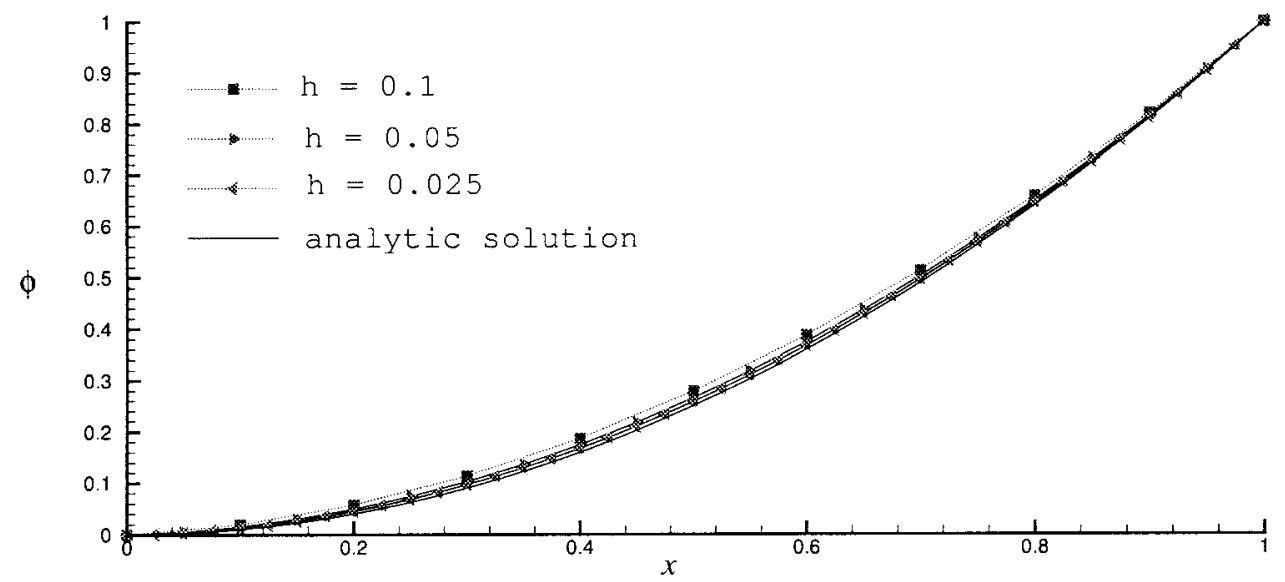

Figure 5. Computed solutions at grids with different resolutions, together with the analytic solution, $\phi=x^{2}$, for the case $(u, K, R)=\left(x, x^{2} / 2,1\right)$ described in the results section. 




Figure 6. Comparison of computed and analytic solutions for the case $(u, K, R, S)=\left(6,1,2,-6 x^{2}-2\right)$ described in the results section.

bounded by $x=0$ and 1 , the analytic solution takes the following form:

$$
\begin{aligned}
\phi(x)= & \exp (3 x)\left[\frac{-7 \exp (-3)+6 \cosh (\sqrt{5})}{\sinh (\sqrt{5})} \sinh (\sqrt{5} x)-6 \cosh (\sqrt{5} x)\right] \\
& +\frac{3}{2} x^{2}+\frac{9}{2} x+\frac{13}{2}
\end{aligned}
$$

In the same domain, $0 \leq x \leq 1$, we also considered the variable coefficient case given by $(u, K, R)=\left(x, x^{2}, 1\right)$. The resulting analytic solution for $S(x)=4 x^{3}$ $-x^{2}$ was obtained as

$$
\phi(x)=x^{2}-x^{3}
$$

As Figures 6 and 7 show, good agreement between analytic and computed solutions is obtained for both cases. This completed our validation analysis, showing the applicability of our scheme to simulate constant/variable model equations with/without source terms.

\section{Two-Dimensional Calculation}

Tests were then run to validate the numerical scheme that has been developed to solve the two-dimensional equation (34). The chosen two-dimensional test is amenable to analytic solution. In the unit square $0 \leq x, y \leq 1$, the solution is 


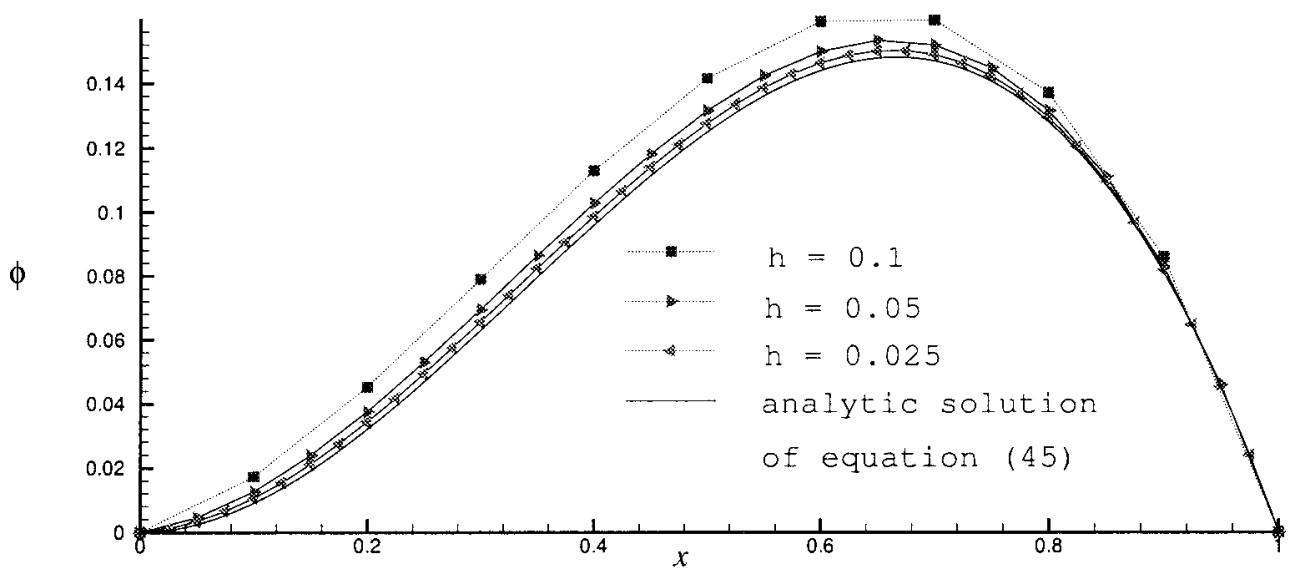

Figure 7. Computed solutions at grids with different resolutions, together with the analytic solution, $\phi=x^{2}-x^{3}$, for the case $(u, K, R, S)=\left(x, x^{2}, 1,4 x^{3}-x^{2}\right)$ described in the results section.

computed under

$$
u=-x, \quad v=-y, \quad K=\sqrt{\frac{8 x+8 x^{3}+3 x y^{2}+5 x^{3} y^{2}}{2\left(x-x^{3}\right)\left(y^{2}+2\right)}},
$$

and $R^{2}=1$. The resulting exact solution takes the following form:

$$
\phi=x\left(1-x^{2}\right)\left(y^{2}+2\right)
$$

We computed solutions on two-dimensional, continuously refined grids. The computed errors are cast in their $L_{2}$ norms and are plotted against grid sizes in Figure 8. As this figure shows, good agreement with the analytic solution is obtained, with a rate of convergence of 1.773 .

\section{CONCLUSIONS}

This article has presented the results of our current efforts in developing a discretization scheme for simulation of the two-dimensional convection-diffusion equation with linear production. The basis for the method presented here is use of the analytic solution in the development of one-dimensional flux discretization scheme. Some progress has been made in extending this analytic formulation to simulate the two-dimensional equation using the operator-splitting ADI scheme. We benchmarked the developed scheme with great success by solving several test problems analytically. 


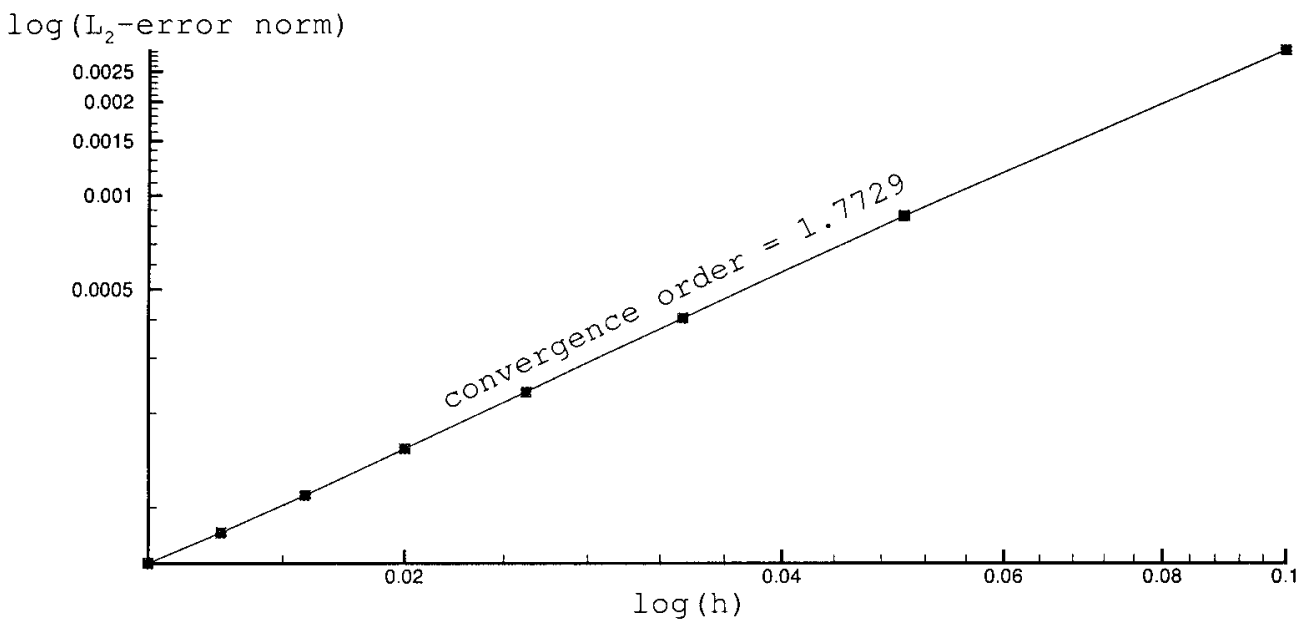

Figure 8. The rate of convergence of the proposed scheme, where $\log \left(L_{2}\right.$ error norms $)$ are plotted against $\log (\Delta x)$. The analytic solution of the test problem is $\phi=x\left(1-x^{2}\right)\left(y^{2}+2\right)$ for the case $(u, v$, $K, R)=\left(-x,-y, \sqrt{\left(8 x+8 x^{3}+3 x y^{2}+5 x^{3} y^{2}\right) / 2\left(x-x^{3}\right)\left(y^{2}+2\right)}, 1\right)$.

\section{REFERENCES}

1. I. Harari and T. J. R. Hughes, Stabilized Finite Element Methods for Steady AdvectionDiffusion with Production, Comput. Meth. Appl. Mech. Eng., vol. 115, pp. 165-191, 1994.

2. I. Harari and T. J. R. Hughes, Finite Element Methods for the Helmholtz Equation in an Exterior Domain: Model Problems, Comput. Meth. Appl. Mech. Eng., vol. 87, pp. 59-96, 1991.

3. L. H. Thomas, Elliptic Problems in Linear Difference Equations over a Network, Watson Scientific Computing Lab. Rep., Columbia University, New York, 1949.

4. R. F. Warming and B. J. Hyette, The Modified Equation Approach to the Stability and Accuracy Analysis of Finite-Difference Methods, J. Comput. Phys., vol. 14, pp. 159-179, 1974. 